\title{
Children with Down Syndrome and Leukemia in Kuwait- Experience of the only Pediatric Hematology Oncology Department in Kuwait
}

\author{
Bourusly $M^{*}$, Obaid MA and Bourhamah $M$ \\ NBK Children's Hospital, Sabah Health District, Kuwait
}

\begin{abstract}
Children with Down syndrome (DS) are at a greater risk of developing leukemia. The aim of this study is to shed light on children with Down syndrome who develop leukemia; the complications and risks they face may lead to their morbidity and mortality. This is a retrospective study of 34 children with Down syndrome who were diagnosed with leukemia at NBK Children's Hospital in Kuwait from 2001-2016. There were 19 patients with acute lymphoblastic leukemia (ALL) and 15 patients with acute myeloid leukemia (AML). Most patients completed chemotherapy and went into remission after the initial induction, despite suffering from many expected complications during the course of treatment. Twenty six patients remained cancer free up till now. Mortality was attributed to sepsis or pneumonia. Children with Down syndrome who were diagnosed with ALL carried worse prognosis than children with Down syndrome who were diagnosed with AML, and those with additional medical problems are at more risk of complications and death.
\end{abstract}

\section{Introduction}

Kuwait has a high incidence of Down syndrome compared to other countries [1-3]. Patients with Down syndrome develop various comorbid conditions including cardiovascular defects, hypothyroidism, craniofacial dysmorphism and learning disabilities [4]. Furthermore, children with Down syndrome have an increased risk of acute leukemia. In fact DS children aged 5 years and younger have 40 fold increased risk of acute lymphocytic leukemia (ALL), and 150 fold increased risk of acute myeloid leukemia (AML) [5]. The majority of DS with AML are AML-M7 subtype or acute megakaryoblastic leukemia [6]. ALL in Down syndrome is 1.7 times more frequent than AML. Most children with Down syndrome develop ALL of a precursor B cell immunophenotype ( $>90 \%$ ) [5]. Most of the mortality of Down syndrome patients with acute leukemia during chemotherapy is attributed to sepsis. It is evident that Down syndrome patients with ALL carry a worse prognosis than Down syndrome patients with AML [3].

\section{Aim and objectives}

This is a retrospective observational study, approved by the ethics committee of the Ministry of Health of Kuwait, of 34 Down syndrome children who were diagnosed with leukemia, and treated at NBK Children's Hospital, Sabah health district in Kuwait. The aim of this study was to determine the outcome of treatment of leukemia in children with Down syndrome in Kuwait. There were four objectives in this study:

- To calculate the number of children with Down syndrome who presented with leukemia, and compare them to healthy Down children and the general population.

- To develop a national registry of children with Down syndrome who developed leukemia.
- To shed light on the complications during treatment and possible risks of morbidity and mortality in order to improve the services provided to these patients.

- To see the effect of improvement of chemotherapy protocols and services on overall survival of these children.

\section{Material and method}

The NBK Children's Hospital at Sabah health district area is the only hospital in Kuwait that manages and treats pediatric leukemia. On average, the hospital receives 46 new cases per year. The incidence of leukemia in Kuwait is 1-2 cases per 100,000 children below the age of 16 years. The hospital was established in the late 1970s as a hematology and leukemia unit following the pediatric department at Al-Sabah hospital, which later became into a department. In the summer of 2017, this department was named a NBK Children's Hospital, an independent hospital that does not follow the Sabah hospital, after the opening of the second modern extension building. Patients' files were retrieved back to year 2001 (the year the permanent filing system was established).

There were 34 patients with Down syndrome who were diagnosed with acute leukemia and referred since 2001. According to a recent survey, there are 3000 people with Down syndrome in Kuwait, at an incidence rate of 1 in 1214 per year [1]; and generally the hospital

Correspondence to: Maha Jassim Bourusly, DCH, MRCPCH, Kuwaiti Board of Pediatrics, NBK Children's Hospital, PO Box: 4078, Kuwait, Tel: 24818219; E-mail: m_bourusly@yahoo.com

Key words: Down syndrome, acute lymphoblastic leukemia, acute myeloid leukemia

Received: August 06, 2017; Accepted: September 04, 2017; Published: September 07, 2017 
receives around two children with Down syndrome per year. The data was collected from the files of these patients with protection of their identities, and after the agreement of the Ministry of Health's ethical committee. Results were analyzed using SPSS software (version 20.0 for Windows). SPSS was used for all statistical analyses for categorical variables.

\section{Results}

There were 34 children with Down syndrome who presented with leukemia at NBK Children's Hospital since 2001; nineteen were boys and fifteen were girls (Table 1). The eldest child at presentation was 14 years old and the youngest was one month old; $76 \%$ of the patients were aged 5 years or younger, with the median age of 2 years. Fifteen patients were Kuwaitis, while the rest were of other nationalities. Most children had other medical problems at presentation such as hypothyroidism or congenital cardiac defects: Eighteen had hypothyroidism at presentation, and 21 children had cardiac defects of different severity. Eight children reported a healthy past history before presentation with leukemia (Table 1).

Nineteen children presented with acute lymphoblastic leukemia (ALL), and 15 with different types of acute myeloid leukemia (Figure 1). Most children received the British chemotherapy protocol UK (Table 2). Only 15 children tolerated full dose of the chemotherapy as per protocol; the dose of chemotherapy was adjusted for the rest of the patients (Table 3) due to life threatening chemotherapy toxicity. Almost all children went into remission after the induction chemotherapy course, and completed the chemotherapy course according to the protocol. Among the children who did not complete the chemotherapy protocol, three died during or just after induction, and one died during re-induction.

Seven children with ALL had hematological relapse of leukemia (Table 4); one of them had hematological and testicular relapse, and one child relapsed twice. None of the children with AML had a relapse so far. Children with ALL who presented with relapse received full dose chemotherapy as per UK-R2 protocol; however, three received half the dose due to toxicity. Among the children who had a relapse, four passed away and one child underwent bone marrow transplant from a matched related donor.

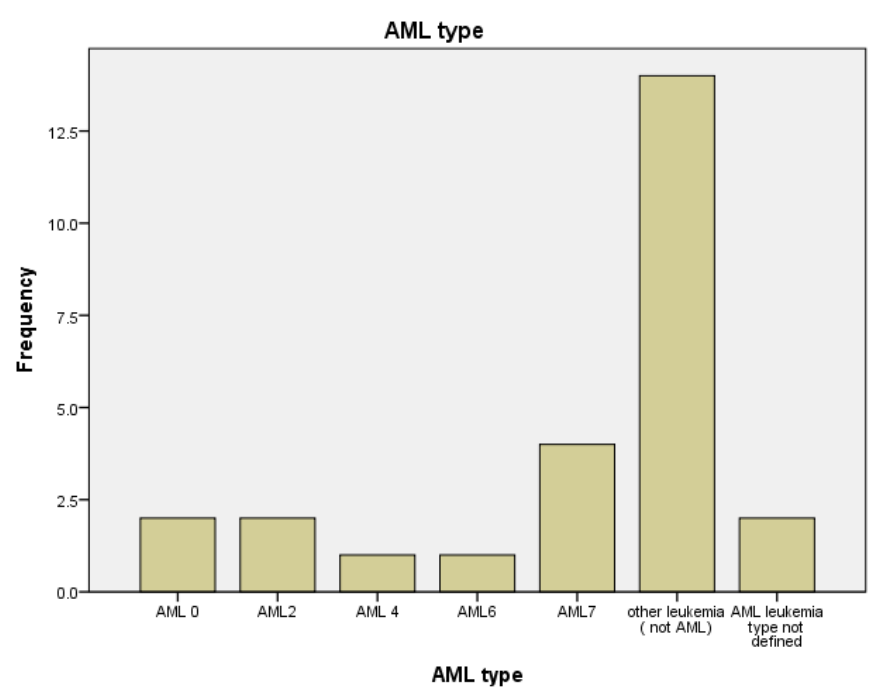

Figure 1. Type of AML
Table 1. Summary of results

\begin{tabular}{|c|c|c|}
\hline Category & $\mathbf{N}=\mathbf{3 4}$ & percentage \\
\hline \multicolumn{3}{|l|}{ Age } \\
\hline$\leq 5 \mathrm{yrs}$ & 26 & $76 \%$ \\
\hline$>5 \mathrm{yrs}$ & $8 / 34$ & $23 \%$ \\
\hline \multicolumn{3}{|l|}{ Sex } \\
\hline Male & $19 / 34$ & $56 \%$ \\
\hline Female & $15 / 34$ & $44 \%$ \\
\hline \multicolumn{3}{|l|}{ Nationality } \\
\hline Kuwaiti & $15 / 34$ & $44 \%$ \\
\hline Non Kuwait & $19 / 34$ & $56 \%$ \\
\hline \multicolumn{3}{|l|}{ Type of leukemia } \\
\hline ALL & $19 / 34$ & $56 \%$ \\
\hline AML & $15 / 34$ & $44 \%$ \\
\hline \multicolumn{3}{|l|}{ Thyroid disease } \\
\hline Hypothyroidism & $18 / 34$ & $53 \%$ \\
\hline Euthyroid & $16 / 34$ & $47 \%$ \\
\hline \multicolumn{3}{|l|}{ Cardiac disease } \\
\hline TOF & $8 / 34$ & $23.50 \%$ \\
\hline ASD & $11 / 34$ & $32.50 \%$ \\
\hline VSD & $1 / 34$ & $2.90 \%$ \\
\hline PPS & $1 / 34$ & $2.90 \%$ \\
\hline Normal & $13 / 34$ & $38.20 \%$ \\
\hline \multicolumn{3}{|l|}{ Relapse } \\
\hline ALL & $7 / 34$ & $20 \%$ \\
\hline AML & $0 / 34$ & $0 \%$ \\
\hline \multicolumn{3}{|l|}{ Mortality } \\
\hline ALL & $6 / 34$ & $17 \%$ \\
\hline AML & $2 / 34$ & $6 \%$ \\
\hline
\end{tabular}

Table 2. Chemotherapy protocols received

\begin{tabular}{|c|c|}
\hline Chemotherapy Protocol & $\mathbf{N}=\mathbf{3 4}$ \\
\hline UK-ALL & 16 \\
\hline UK-AML & 15 \\
\hline ccg-1952 & 1 \\
\hline BFM protocol & 1 \\
\hline other & 1 \\
\hline Total & 34 \\
\hline
\end{tabular}

Most children had chemotherapy toxicity related complications during their therapy, such as pancytopenia and febrile neutropenia as well as central line complications. Eight children died due to infection: six children with ALL and two children with AML (Tables 5 and 6). Among the children who died, three were receiving full dose of chemotherapy, four children were receiving half dose, and one child was receiving $25 \%$ of dose (Table 7 ).

Eighteen children had hypothyroidism which was either diagnosed before, during or after completion of the treatment. Eight children had respiratory compromise; one child was diagnosed with pulmonary $\mathrm{TB}$ few months before the diagnosis of leukemia (Table 8).

Most mothers of these children were said to be healthy at diagnosis (25 mothers), as well as fathers (25 fathers). Those who were not healthy were complaining of hypertension, diabetes mellitus or other medical problems. There was no history of cancer in 22 families. Most children had 3-4 siblings; one child had another sibling with Down syndrome who did not develop leukemia so far.

Children aged less than 3 years are more likely to have AML than ALL $(\mathrm{P}=0.002)$, and children older than 3 years are more likely to have ALL. Children with AML showed better response to treatment and survival (mortality was 16\%), in comparison to children with 
Table 3. Chemotherapy dose adjustment

\begin{tabular}{|c|c|c|}
\hline Dose & $\mathbf{N = 3 4}$ & Percentage \\
\hline Full dose & 15 & $44.1 \%$ \\
\hline $75 \%$ of dose & 3 & $8.8 \%$ \\
\hline $50 \%$ of dose & 14 & $41.2 \%$ \\
\hline $25 \%$ of dose & 2 & $5.9 \%$ \\
\hline Total & 34 & \\
\hline
\end{tabular}

Table 5. Causes of death in Down syndrome children with leukemia

\begin{tabular}{|c|c|c|c|c|c|c|}
\hline \multicolumn{2}{|c|}{} & \multicolumn{4}{|c|}{ Cause of death } & \multirow{2}{*}{ Total } \\
\cline { 3 - 7 } \multicolumn{2}{|c|}{} & Alive & Septicemia & Pneumonia & Others & \\
\hline \multirow{3}{*}{ Viability } & Alive & 26 & 0 & 0 & 0 & 26 \\
\cline { 2 - 7 } & Dead & 0 & 4 & 3 & 1 & 8 \\
\hline \multicolumn{2}{|c|}{ Total } & 26 & 4 & 3 & 1 & 34 \\
\hline
\end{tabular}

Table 6. Mortality and type of leukemia

\begin{tabular}{|c|c|c|c|c|}
\hline \multicolumn{2}{|c|}{} & \multicolumn{2}{|c|}{ Diagnosis of leukemia } & Total \\
\cline { 2 - 5 } & AML & ALL & \\
\hline \multirow{3}{*}{ Cause of death } & Alive & 12 & 14 & 26 \\
\cline { 2 - 5 } & Septicemia & 1 & 3 & 4 \\
\cline { 2 - 5 } & Pneumonia & 0 & 3 & 3 \\
\cline { 2 - 5 } & Others & 1 & 0 & 1 \\
\cline { 2 - 5 } & & 14 & 20 & 34 \\
\hline
\end{tabular}

Table 4. Relapse down syndrome children with leukemia

\begin{tabular}{|c|c|c|c|c|}
\hline & & \multicolumn{2}{|c|}{ Diagnosis of leukemia } & \multirow{2}{*}{ Tota } \\
\hline & & AML & ALL & \\
\hline \multirow{2}{*}{ Relapse } & Yes & 0 & 7 & 7 \\
\hline & No & 14 & 13 & 27 \\
\hline \multicolumn{2}{|c|}{ Total } & 14 & 20 & 34 \\
\hline
\end{tabular}

Table 7. The cause of death and chemotherapy dose received

\begin{tabular}{|c|c|c|c|c|c|c|}
\hline \multicolumn{2}{|c|}{} & \multicolumn{5}{|c|}{ Dose of the chemotherapy } \\
\hline \multirow{2}{*}{} & Full dose & $\begin{array}{c}\mathbf{7 5 \%} \text { of } \\
\text { dose }\end{array}$ & Half dose & $\begin{array}{c}\mathbf{2 5 \%} \text { of } \\
\text { dose }\end{array}$ & Total \\
\hline Alive & Alive & 12 & 3 & 10 & 1 & 26 \\
\hline \multirow{2}{*}{$\begin{array}{c}\text { Dead and } \\
\text { the Cause } \\
\text { of death }\end{array}$} & Septicemia & 2 & 0 & 1 & 1 & 4 \\
\cline { 2 - 8 } & Pneumonia & 1 & 0 & 2 & 0 & 3 \\
\hline Total & Others & 0 & 0 & 1 & 0 & 1 \\
\hline
\end{tabular}

Table 8. Other medical problem DS children face

\begin{tabular}{|c|c|c|}
\hline Medical problem & $\mathbf{N = 3 4}$ & Percentage \\
\hline Hypothyroidism & 18 & $53 \%$ \\
\hline Cardiac lesion & 30 & $88 \%$ \\
\hline $\begin{array}{c}\text { Intestinal lesion/abdominal } \\
\text { defects }\end{array}$ & 6 & $17 \%$ \\
\hline Respiratory disease & 8 & $23 \%$ \\
\hline Diabetes mellitus & 1 & $3 \%$ \\
\hline Multiple medical problems & 17 & $50 \%$ \\
\hline Healthy & 8 & $23 \%$ \\
\hline
\end{tabular}

ALL (mortality was $31 \%$ ). Children with AML could not tolerate full dose of chemotherapy as normal children, and the dose had to be reduced to avoid complications in two thirds of children, specifically for younger children. Dose adjustment was in accordance with Down syndrome guideline recommendations in the chemotherapy protocol, and according to patients' general condition and treatment tolerability. Dose adjustment had no direct effect on survival.

The type of leukemia affected the probability of relapse $(p=0.013)$ as patients with ALL were more likely to have a relapse than AML.
Those who had respiratory problem had higher risk of death $(\mathrm{p}=0.027)$; however, they did not have higher risk for disease relapse ( $\mathrm{p}=0.173)$.

Parents' health did not increase the risk of death. Parents' age at diagnosis and their health state has no direct effect on mortality or survival.

\section{Discussion}

Kuwait has a high incidence of Down syndrome, when compared to other countries [3], this might be due to multiple factors such as mothers continue to have children beyond the forth decade of life, restriction of pregnancy termination, and increased number of children per family. A child with Down syndrome is liable to have multiple medical problems as he or she grows up. Children with Down syndrome are 10 to 20 fold more prone to develop acute lymphoblastic leukemia or acute myeloid leukemia in comparison to non-Down syndrome children $[7,8]$. In this study 1 in 100 DS developed childhood leukemia, which is underrepresented incidence as we do not have the incidence of leukemia for adults with DS.

There is an increased chance of morbidity in children with Down syndrome and leukemia as many children already have congenital heart disease, respiratory problems or gastrointestinal problems $[9,10]$. Treatment of a Down syndrome child with leukemia can be quite challenging as they will face two major complications during treatment: myelosuppression and chemotherapy-related toxicity. The majority of children with Down syndrome will develop myelosuppression as a result of the chemotherapy treatment received, and they are likely to suffer from recurrent infections such as pneumonia, line infection, cellulitis and other skin infections [11]. None of the children involved in this study died of the disease per se; $23 \%$ of children in this study died due to sepsis or infections as a result of immunosuppression caused by chemotherapy treatment they received [12].

DS children with ALL seem to have higher mortality, and this has been demonstrated clearly in this study. The majority of deaths occurred during or just after induction chemotherapy, most likely due to the intensive chemotherapy rendering them vulnerable to severe neutropenia and infections $[2,12]$. There is an increased methotrexateassociated toxicity in DS ALL patients. The high event-free survival (EFS) rates of DS AML patients and in particular, patients with megakaryocytic leukemia (AMkL), at least in part reflects an increased sensitivity to cytosine arabinoside (ara-C) secondary to increased expression of the chromosome 21-localized gene, which increase the susceptibility of cells to undergo apoptosis [13]. AMkL is the most common French-American-British subtype of DS AML patients, as reported to represent more than $90 \%$ of DS AML cases. The majority of cases are diagnosed before the age of 4 years [14,15], and uniformly harbor somatic mutations in the transcription factor GATA1 gene [16]; however we have seen DS children with different types of AML in this study. The other AML French-American-British subtypes described in DS AML including M0, M1/M2, and M6 [17]. In this study, most patients were diagnosed as AML- M7; however other types of AML were seen such as M0, M2, M4 and M6. As expected, children with AML in this study coped better than those with ALL, and were less prone to relapse than ALL.

All children in this study who had either hematological or testicular relapse were diagnosed with ALL as their primary diagnosis, and relapsed to their original disease, in exception to one child who relapsed with AML [18]; however we cannot generalize this fact due to small sample size in this study. Regarding the toxicity of the 
chemotherapy treatment, it was noticed that receiving half the dose to avoid chemotherapy toxicity may not increase the risk of death among children with ALL, or their relapse risk. It may probably decrease morbidity in DS children [19,20].

Cardiac studies performed during or post treatment showed that most patients had normal left ventricular ejection fraction; however, there were no records available for seven patients. We definitely need series of cardiac studies to see the overall toxic effect of chemotherapy on these children before, during and after the treatment, and whether there is a link between having a cardiac disease and developing leukemia in Down syndrome [21]. Recently, our protocols have been modified in accordance with international protocols in order to avoid the toxicity in these children, the effect would be observed in future; though this study has shown that the reduced dose protocol did not increase the rate of relapse as shown in other studies [19,22].

Children who had other medical problems, such as those with diabetes mellitus or sleep apnea, were more prone to death as seen in this study. Perhaps those children had additional factors to the development of sepsis.

The prevalence of thyroid disease in children with Down syndrome is known to be higher than non-Down syndrome children [23].

Parents' health was not a factor in the morbidity and mortality of these children. Most families had on average 3-4 children.

All children were diagnosed and referred by the pediatricians in the hospital of their health districts, after showing symptoms and signs of the disease. The youngest child presented to our department was one month old. There is no current pathway to follow children with Down syndrome hematologically in Kuwait [24]; i.e. to do GATA1 test at infancy in children with abnormal hemogram, to filter the cases suspected to have leukemia or transient leukemia (TL) [23,25], as somatic mutations of GATA1 are found in almost all cases of Down syndrome or mosaic trisomy 21 associated TL and AMKL [26]. All the focus now is on the other medical problems the DS child is facing. Currently, a proposal to address this issue has been discussed to be applied soon.

One family had two children with Down syndrome, one of them has leukemia. They coped well with the disease, yet we do not know the quality of life of this family as this was out of the scope of this study [27].

\section{Conclusion}

Management of a child with Down syndrome and leukemia can be challenging, and need special care, and follow up to avoid complications. More effort should be addressed to follow DS children with abnormal hemogram during early infancy and beyond. In this study a national registry was created for DS children with leukemia, as well as in depth focus on the complications faced during treatment that might lead to possible risks of morbidity and mortality was addressed in order to improve the services provided to these patients. The treatment protocols and management evolved with time; however, the effect of improvement of chemotherapy protocols and services on overall survival of these children yet to be seen.

\section{Limitations}

In this study, the sample included only DS children with leukemia. Though the advantage of having all the children with DS with leukemia treated in one center in Kuwait; it would be more beneficial to have adult DS statistics to be added in order to calculate the prevalence and incidence and event free survival rates in DS patients in Kuwait,
We do not know the full impact of the disease on the family: socially, psychologically and economically, as this was out of the scope of this study.

This study was designed with the intention to shed the light on the problems encountered during the treatment of DS children with leukemia. However, due to the small sample size, we tried to include all the children, though they received different versions of the chemotherapy protocols designated to treat ALL, i.e children with ALL allocated to four different ALL protocols: UK -ALL 1999, 2003, CCG-1952 BFM protocol. This happened for two reasons: the first was different versions of protocols evolved with time and the other reason was that some children received their initial chemotherapy protocol abroad.

\section{Recommendation for those who will write in future}

Although this retrospective study had shed the light on the problem of DS children with leukemia face during treatment in Kuwait, we need further studies such as cohort or perhaps quality of life study to see the long term effects of chemotherapy on DS children.

\section{Declaration}

The authors has nothing to declare.

\section{Acknowledgment}

The authors would like to thank Mr. Ahmed Al-Musaileem and Miss. Suad Alanzi for their assistance in the this study.

\section{References}

1. 3000 cases for Down syndrome in Kuwait- Alqabas electronic. http://alqabas $\mathrm{com} / 373209$ /

2. Al-Awadi S, et al. Down syndrome in Kuwait: an epidemiology approach. Medics ME.

3. Jastaniah W, Alsultan A, Al Daama S, Ballourah W, Bayoumy M, et al. (2017) Treatment results in children with myeloid leukemia of Down syndrome in Saudi Arabia: A multicenter SAPHOS leukemia group study. Leuk Res 58: 48-54. [Crossref]

4. Khan I, Malinge S, Crispino J (2011) Myeloid leukemia in Down syndrome. Crit Rev Oncog 16: 25-36. [Crossref]

5. Webb D, Roberts I, Vyas P (2007) Haematology of Down syndrome. Arch Dis Child Fetal Neonatal Ed 92: F503-F507. [Crossref]

6. Rabin KR, Whitlock JA (2009) Malignancy in children with trisomy 21. Oncologist 14 164-173. [Crossref]

7. Xavier AC, Yubin Ge, Taub JW (2009) Down syndrome and Malignancies: A Unique Clinical Relationship. A Paper from the 2008 William Beaumont Hospital Symposium on Molecular Pathology. J Mol Diagn 11: 371-380. [Crossref]

8. Taub JW (2001) Relationship of chromosome 21 and acute leukemia in children with Down syndrome. J Pediatr Hematol Oncol 23: 175-178. [Crossref]

9. Maloney KW (2011) Acute lymphoblastic leukaemia in children with Down syndrome: an updated review. Br J Haematol 155: 420-425. [Crossref]

10. Salazar EG, Li Y, Fisher BT, Rheingold SR, Fitzgerald J, et al. (2016) Supportive care utilization and treatment toxicity in children with Down syndrome and acute lymphoid leukaemia at free-standing paediatric hospitals in the United States. Br J Haematol 174(4): 591-599. [Crossref]

11. Rabin KR, Smith J, Kozinetz CA(2011) Myelosuppression and infectious complications in children with Down syndrome and acute lymphoblastic leukemia. Pediatr Blood Cancer.

12. Ceppi F, Stephens D, den Hollander BS3, Krueger J1, Whitlock J1, et al. (2016) Clinical presentation and risk factors of serious infections in children with Down syndrome treated for acute lymphoblastic leukemia. Pediatr Blood Cancer 63: 19491953. [Crossref]

13. Taub JW, Ge Y (2005) Down syndrome, drug metabolism and chromosome 21. Pediatr Blood Cancer 44: 33-39. [Crossref] 
14. Pine SR, Guo Q, Yin C, Jayabose S, Druschel CM, et al. (2007) Incidence and clinical implications of GATA1 mutations in newborns with Down syndrome. Blood 110: 21282131. [Crossref]

15. Ravindranath Y, Abella E, Krischer JP, Wiley J, Inoue S, et al. (1992) Acute myeloid leukemia (AML) in Down's syndrome is highly responsive to chemotherapy: experience on Pediatric Oncology Group AML Study 8498. Blood 80: 2210-2214. [Crossref]

16. Saida S (2016) Evolution of myeloid leukemia in children with Down syndrome. Int $J$ Hematol 103: 365-372. [Crossref]

17. Xavier AC, Ge Y, Taub J (2010) Unique clinical and biological features of leukemia in Down syndrome children. Expert Rev Hematol 3: 175-186. [Crossref]

18. Lange BJ, Kobrinsky N, Barnard DR, Arthur DC, Buckley JD, et al. (1998) Distinctive demography, biology, and outcome of acute myeloid leukemia and myelodysplastic syndrome in children with Down syndrome: children's Cancer Group Studies 2861 and 2891. Blood 91: 608-615. [Crossref]

19. Tomizawa D, Endo A, Kajiwara M, et al. Acute lymphoblastic leukemia in patients with Down syndrome with a previous history of acute myeloid leukemia. Pediatr Blood Cancer 64 .

20. Creutzig U, Reinhardt D, Diekamp S, Dworzak M, Stary J, et al. (2005) AML patients with Down syndrome have a high cure rate with AML-BFM therapy with reduced dose intensity. Leukemia 19: 1355-1360. [Crossref]
21. Uffmann M, Rasche M, Zimmermann M, von Neuhoff C, Creutzig U, et al. (2017) Therapy reduction in patients with Down syndrome and myeloid leukemia: the international ML-DS 2006 trial. Blood 129: 3314-3321. [Crossref]

22. Carozza SE, Bae H, Meath T, Branscum A, Bovbjerg ML, et al. (2016) Evaluation of maternal health and labor and delivery conditions as risk factors for childhood leukemias in children with Down syndrome. Cancer Epidemiol 46: 36-41. [Crossref]

23. Taub JW, Berman JN, Hitzler JK, Sorrell AD, Lacayo NJ, et al. (2017) Improved outcomes for myeloid leukemia of Down syndrome: a report from the Children's Oncology Group AAML0431 trial. Blood 129: 3304-3313. [Crossref]

24. Alford KA, Reinhardt K, Garnett C, Norton A, Böhmer K, et al. (2011) Analysis of GATA1 mutations in Down syndrome transient myeloproliferative disorder and myeloid leukemia. Blood 118: 2222-2238. [Crossref]

25. Gamis AS, Alonzo TA, Gerbing RB, Hilden JM, Sorrell AD, et al. (2011) Natural history of transient myelo-proliferative disorder clinically diagnosed in Down syndrome neonates: a report from the Children's Oncology Group Study A2971. Blood 118: 6752-6759. [Crossref]

26. Williams BA, Meyn MS, Hitzler JK (2011) Transient leukemia in newborns without down syndrome: diagnostic and management challenges. J Pediatr Hematol Oncol 33: e261-e263.

27. Schultz KA, Chen L, Kunin-Batson A, Chen Z, Woods WG, et al. (2017) Health-related Quality of Life (HR-QOL) and Chronic Health Conditions in Survivors of Childhood Acute Myeloid Leukemia (AML) with Down Syndrome (DS): A Report From the Children's Oncology Group. J Pediatr Hematol Oncol 39: 20-25. [Crossref]

Copyright: (C2017 Bourusly M. This is an open-access article distributed under the terms of the Creative Commons Attribution License, which permits unrestricted use, distribution, and reproduction in any medium, provided the original author and source are credited. 